\title{
SIMULATED ANNEALING WITH TSALLIS WEIGHTS FOR $\pm J$ SPIN GLASS
}

\author{
J. KŁOS ${ }^{a, b}$ AND S. KOBE ${ }^{b}$ \\ ${ }^{a}$ Institut für Theoretische Physik, Technische Universität Dresden \\ 01062 Dresden, Germany \\ ${ }^{b}$ Instytut Fizyki, Uniwersytet im. A. Mickiewicza, Coll. Physicum \\ Umultowska 85, 61-614 Poznań, Poland
}

\begin{abstract}
Recently, on the basis of the Tsallis distribution of states the generalized Monte Carlo acceptance probabilities have been proposed and applied to various optimization problems. In the paper we used the generalized simulated annealing algorithms for the $\pm J$ spin glass model. Efficiency of the new annealing procedures was compared with that of the conventional methods. This was done with respect to the number of annealing steps and to the lowest energy found. The algorithms were applied to a system of $70 \times 70$ spins on a square lattice with periodical boundary conditions whose ground state configurations are known exactly. This enables us to evaluate the efficiency in a quantitative way.
\end{abstract}

PACS numbers: $05.50 .+\mathrm{q}, 64.60 . \mathrm{Cn}, 75.10 . \mathrm{Hk}$

Simulated annealing (SA) is a computational tool to deal with hard optimization problems [1]. The method proceeds in such a way that at each annealing step $n$ the temperature $T(n)$ is given and the system is allowed to walk on its phase space long enough so that it will thermalize at $T(n)$. Then the temperature is decreased according to a given annealing schedule and the process is repeated till the final temperature is sufficiently low. Some of the recent applications of SA $[2,3]$ have been based on the Tsallis statistics which unlike the usual Boltzmann-Gibbs theory can be used for nonextensive systems $[4,5]$.

The paper is organized as follows. We first recall the generalized simulated annealings (SAs) based on the Tsallis formalism. Next, we describe the system considered and the simulations. The exact knowledge of the system ground-state energy enables us not only to examine the time and energy characteristics of the generalized SAs but also to check their efficiency quantitatively.

The Tsallis generalized acceptance probability (AP) is defined as

$$
p=\min \left\{1,[1-(1-q) \beta \Delta E]^{\frac{1}{(1-q)}}\right\},
$$

where $\Delta E=E_{\text {new }}-E_{\text {old }}\left(E_{\text {new }}\right.$ and $E_{\text {old }}$ are energies after and before a random reconfiguration) and $q$ is any real number [5]. For $q \rightarrow 1$ the AP of canonical SA 
$p=\min [1, \exp (-\beta \Delta E)]$ is recovered. Since Eq. (1) does not obey detailed balance, Andricioaei and Straub have recently replaced it by another AP [3]

$$
p=\min \left\{1,\left[\frac{1-(1-q(T)) \beta E_{\text {new }}}{1-(1-q(T)) \beta E_{\text {old }}}\right]^{\frac{q(T)}{1-q(T)}}\right\}, \quad \lim _{T \rightarrow 0} q(T)=1 .
$$

In Eq. (1) $q$ is kept constant during a run while in (2) it is a monotonically decreasing function of temperature $T$. Starting with the initial value of $q=q_{\mathrm{i}}$ at the initial temperature $T_{\mathrm{i}}, q$ must tend to one with $T$ going to the final value $T_{\mathrm{f}}$.

We have tested the above SAs for the 2D Edward-Anderson spin glass system of $70 \times 70$ spins whose ground state energy is known exactly [6]. Ising spins are located at each site of a square lattice with periodical boundary conditions. The ferromagnetic and antiferromagnetic bonds $J_{i j}= \pm 1$ between the nearest neighbouring sites are distributed randomly and uniformly so that the fraction of each of them is $c=0.5$. The system energy is

$$
E=-\sum_{i<j} J_{i j} S_{i} S_{j}+2 N
$$

where $S_{i}, S_{j}= \pm 1$ are Ising spins, $N$ is their total number, and the sum on the right hand side runs over the nearest neighbouring sites. The last term in (3) is a shift in the energy scale so that it becomes non-negative [7]. The SAs are tested by applying Monte Carlo (MC) algorithms within single-spin dynamics [8]. $T$ (and $q$ ) are kept constant for $100 \mathrm{~N}$ trial reconfigurations or for $10 \mathrm{~N}$ successful rearrangements, whichever comes first [2]. For the cooling schedules $T_{\mathrm{i}}$ is lowered according to $T=T_{\mathrm{i}}\left(T_{\mathrm{f}} / T_{\mathrm{i}}\right)^{n / N_{\mathrm{a}}}$, where $n=0,1,2, \ldots, N_{\mathrm{a}}$, and $N_{\mathrm{a}}$ is the number of annealing steps. In Eq. (2) $q$ is also decreased exponentially to one, starting from values higher than one [3]. The AP (1) is set to zero whenever the argument of the power law acceptance function is negative.

We have performed 20 independent runs of $N_{\mathrm{a}}=1000$ steps for each of the APs with different values of the other parameters. In terms of the temperature (Fig. 1) low energies are approached faster with (1) for $q<1$. It is particularly the case for $q<0$. However, the disadvantage is that the minimizing stops at higher temperatures and the system is trapped in a set of isoenergetic states. For $q$ slightly smaller than one there is no major difference from the regular SA, and for $q>1$ no energy decrease can be found at all. The SA (2) behaves entirely different. Only for $q_{\mathrm{i}}$ extremely close to one the annealing looks similar to the traditional one though it is a bit slower. In most of the SA steps for all other cases, the energy fluctuates around a constant value and drops rapidly at the end. No special advantage over the traditional SA can be observed in this approach. Similar results are obtained with respect to the number of MC steps per spin (Fig. 2) (one MC step per spin is defined as a sweep over the whole lattice). Equation (1) leads to low-energy states extremely fast especially for $q<0$. However, an immediate trapping of the system in its local energy minima is present. The algorithm (2) needs much more time so that the system can find the low-energy states and for $q_{\mathrm{i}}$ nearly one can only be as fast as the regular $\mathrm{SA}$.

Apart from the temperature and time characteristics of the SAs we are interested in their efficiency, i.e. the energies found at the end of each run (Table). 


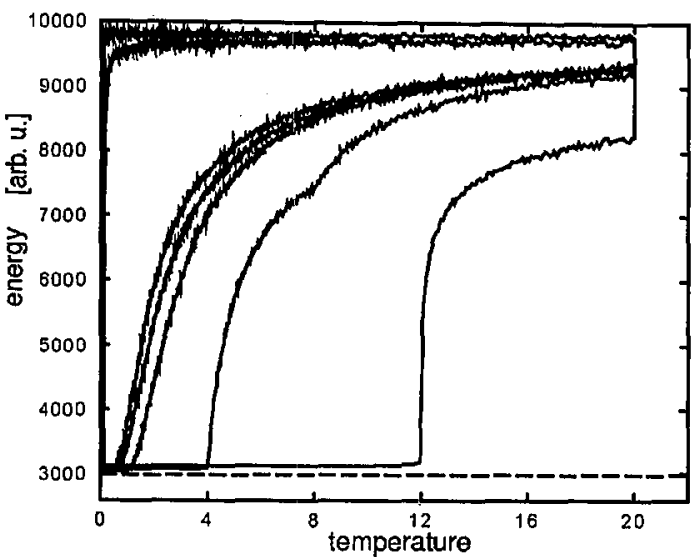

Fig. 1. The energy $E$ vs. temperature $T$ for the average of 20 runs. A $70 \times 70$ spin system with the ground-state energy of 2976 (dashed line) is used. $T_{\mathrm{i}}=20, T_{\mathrm{f}}=0.1$ (in units of $k T$ ). From the bottom right hand side corner to the top left hand side one: AP (1) with $q=-2,0,0.8$, the standards SA $(q=1)$, and AP (2) with $q_{\mathrm{i}}=1.0001,1.01$, 2 , respectively. In case of AP (1) the energy decreases very rapidly even in the "zeroth" annealing step.

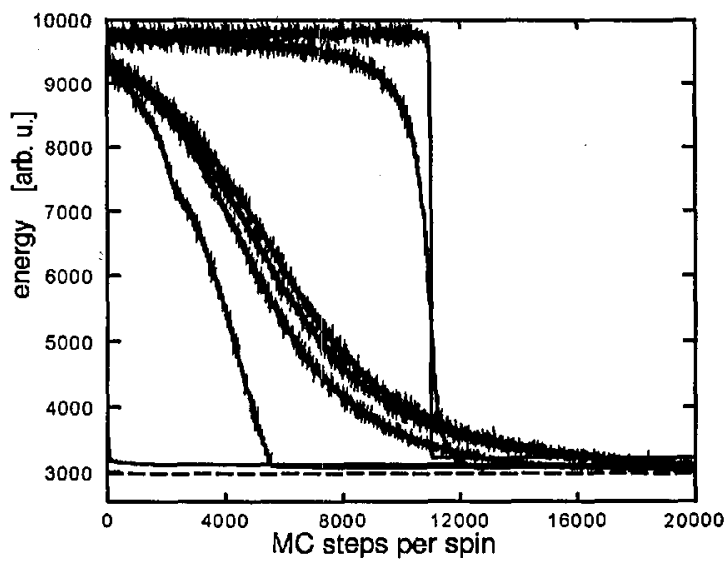

Fig. 2. The energy $E$ versus $M C$ steps per spin for the average of 20 runs for the same system as in Fig. 1. The same order as in Fig. 1 but from the bottom left hand side corner to the top right hand side one.

Using (1) we can see from Table (part (a)) that the closer to one $q$ is, the lower are both the lowest final energy $E_{l}$ and its average $\left\langle E_{l}\right\rangle$. However, the results are not qualitatively better than those obtained using the standard scheme (b). For $q=-2$ the algorithm is indeed very fast but simultaneously the system is trapped in a subspace of states with relatively high energies. When applying (2), for $q_{\mathrm{i}}$ slightly larger than one we have arrived at relatively low final energies (c). Although some of the $E_{l}$ values are even a little lower than those in (b) we do 
TABLE

The same system as in Fig. 1. The lowest final energy $E_{l}$ out of 20 independent runs found for each pair of $T_{\mathrm{i}}$ and $T_{\mathrm{f}}$ using: (a) AP (1), (b) standard SA, (c) AP (2). $\left\langle E_{l}\right\rangle$ is the average over the 20 runs of $E_{l}$ obtained in each of them.

\begin{tabular}{c|c|c|c|c|c|c|c|c|c|c}
\hline \hline \multicolumn{5}{c|}{ (a) } & \multicolumn{3}{c|}{ (b) } & \multicolumn{3}{c}{ (c) } \\
\hline$T_{\mathrm{i}}$ & $T_{\mathrm{f}}$ & $q$ & $E_{l}$ & $\left\langle E_{l}\right\rangle$ & $q$ & $E_{l}$ & $\left\langle E_{l}\right\rangle$ & $q_{\mathrm{i}}$ & $E_{l}$ & $\left\langle E_{l}\right\rangle$ \\
\hline 20 & 0.1 & 0.8 & 3012 & 3032.00 & 1 & 3008 & 3026.20 & 2 & 3152 & 3217.80 \\
10 & 0.1 & 0.8 & 3012 & 3027.00 & 1 & 3012 & 3024.80 & 1.01 & 3044 & 3066.00 \\
20 & 0.1 & 0 & 3044 & 3069.89 & 1 & & & 1.01 & 3036 & 3060.60 \\
10 & 0.1 & -2 & 3096 & 3122.67 & 1 & & & 1.0001 & 3004 & 3020.60 \\
10 & 0.001 & -2 & 3096 & 3124.20 & 1 & 3020 & 3031.4 & 1.0001 & 3012 & 3025.80 \\
20 & 0.1 & -2 & 3092 & 3125.33 & 1 & & & 1.0001 & 3008 & 3024.40 \\
20 & 0.001 & -2 & 3076 & 3119.40 & 1 & 3008 & 3031.4 & 1.0001 & 3004 & 3030.00
\end{tabular}

not think that the difference is sufficient to treat this AP as more efficient. The tendency is such that for larger $q_{\mathrm{i}}$ both $E_{l}$ and $\left\langle E_{l}\right\rangle$ are higher as well. None of the algorithms has found the true ground state whose energy was 2976.

Summarizing, none of the generalized SAs has proven qualitatively more efficient in the above optimization problem. Using them we have arrived at states whose energies compare with those found with the standard MC method. However, in terms of very quick but imperfect minimization the AP (1) seems the most useful. Our results together with others confirm the general opinion that the methods are strongly model dependent.

We acknowledge support from GK "Struktur- und Korrelationseffekte in Festkörpern". We thank J. Weißbarth and M. Nogala for helpful discussions and the authors of [6] for allowance to use their computer data.

\section{References}

[1] S. Kirkpatrick, C.D. Gelatt, Jr., M.P. Vecchi, Science 220, 671 (1983).

[2] T.J.P. Penna, Phys. Rev. E 51, R1 (1995).

[3] I. Andricioaei, J.E. Straub, Phys. Rev. E 53, R3055 (1996).

[4] C. Tsallis, J. Stat. Phys. 52, 479 (1988).

[5] D.A. Stariolo, C. Tsallis, in: Annual Reviews of Computational Physics II, Ed. D. Stauffer, World Scientific, Singapore 1995, p. 343.

[6] C. De Simone, M. Diehl, M. Jünger, P. Mutzel, G. Reinelt, G. Rinaldi, J. Stat. Phys. 80, 487 (1995).

[7] I. Andricioaei, J.E. Straub, Physica A 247, 553 (1997).

[8] K. Binder, D.W. Herrmann, Monte Carlo Simulation in Statistical Physics, in series: Springer Series in Solid-State Sciences, Vol. 80, Springer-Verlag, Berlin 1988. 